\title{
Maternal Probiotic or Synbiotic Supplementation Modulates Jejunal and Colonic Antioxidant Capacity, Mitochondrial Function, and Microbial Abundance in Bama Mini-piglets
}

\author{
Kai Wang $\mathbb{D},{ }^{1,2,3,4}$ Xiangfeng Kong $\mathbb{D}^{2},{ }^{2}$ Md. Abul Kalam Azad $\mathbb{D},{ }^{2}$ Qian Zhu, ${ }^{2}$ Liang Xiong, \\ Yuzhong Zheng $\mathbb{D}^{4}{ }^{4}$ Zhangli Hu, ${ }^{1,3}$ Yulong Yin, ${ }^{2}$ and Qinghua He ${ }^{1}$ \\ ${ }^{1}$ Department of Food Science and Engineering, College of Chemistry and Environmental Engineering, Shenzhen University, \\ Shenzhen 518000, China \\ ${ }^{2}$ CAS Key Laboratory of Agro-Ecological Processes in Subtropical Regions, Hunan Provincial Key Laboratory of Animal Nutritional \\ Physiology and Metabolic Process, National Engineering Laboratory for Pollution Control and Waste Utilization in Livestock and \\ Poultry Production, Institute of Subtropical Agriculture, Chinese Academy of Sciences, Changsha 410125, China \\ ${ }^{3}$ Key Laboratory of Optoelectronic Devices and Systems of Ministry of Education and Guangdong Province, College of \\ Optoelectronic Engineering, Shenzhen University, Shenzhen 518000, China \\ ${ }^{4}$ School of Food Engineering and Biotechnology, Hanshan Normal University, Chaozhou 521041, China
}

Correspondence should be addressed to Xiangfeng Kong; nnkxf@isa.ac.cn and Qinghua He; qinghua.he@szu.edu.cn

Received 3 December 2020; Revised 6 March 2021; Accepted 25 March 2021; Published 5 May 2021

Academic Editor: Julia M. Dos Santos

Copyright (c) 2021 Kai Wang et al. This is an open access article distributed under the Creative Commons Attribution License, which permits unrestricted use, distribution, and reproduction in any medium, provided the original work is properly cited.

\footnotetext{
The present study was conducted to investigate the effects of maternal probiotic or synbiotic supplementation during gestation and lactation on antioxidant capacity, mitochondrial function, and intestinal microbiota abundance in offspring weaned piglets. A total of 64 pregnant Bama mini-sows were randomly allocated into the control group (basal diet), antibiotic group (basal diet $+50 \mathrm{~g} / \mathrm{t}$ virginiamycin), probiotic group (basal diet $+200 \mathrm{~mL} / \mathrm{d}$ probiotics per pig), or synbiotic group (basal diet $+200 \mathrm{~mL} / \mathrm{d}$ probiotics per pig $+500 \mathrm{~g} / \mathrm{t}$ xylo-oligosaccharides). On day 30 of post-weaning, eight piglets per group with average body weight were selected for sample collection. The results showed that maternal probiotic supplementation increased the catalase (CAT) activity in plasma and glutathione peroxidase (GSH-Px) and superoxide dismutase (SOD) activities in plasma, jejunum, and colon of piglets while decreased the malondialdehyde (MDA) and $\mathrm{H}_{2} \mathrm{O}_{2}$ concentrations in plasma compared with the control group $(P<0.05)$. Moreover, maternal synbiotic supplementation increased the plasma CAT activity, jejunal glutathione and GSH-Px activities, jejunal and colonic total antioxidant capacity activity, and plasma and colonic SOD activity while decreased the colonic MDA concentration of offspring piglets compared with the control group $(P<0.05)$. The mRNA levels of antioxidant enzyme-related genes (copper- and zinc-containing superoxide dismutase, nuclear factor erythroid 2-related factor 1, and nuclear factor erythroid 2-related factor 2) and mitochondrial-related genes (adenosine triphosphate synthase alpha subunit, adenosine triphosphate synthase $\beta$, and mitochondrial transcription factor $A$ ) in the jejunal mucosa were significantly upregulated, while the level of colonic peroxisome proliferator-activated receptor $\gamma$ coactivator-1 $\alpha$ was downregulated by maternal synbiotic supplementation $(P<0.05)$. Maternal probiotic supplementation increased $(P<0.05)$ the Bacteroidetes abundance in the jejunum and Bifidobacterium abundance in the jejunum and colon, and synbiotic supplementation increased $(P<0.05)$ the abundances of Firmicutes, Bacteroidetes, Bifidobacterium, and Lactobacillus in the jejunum of piglets. Furthermore, correlation analysis revealed that intestinal microbiota abundances were significantly correlated with antioxidant enzyme activities and mitochondrial-related indexes. These findings indicated that maternal probiotic or synbiotic supplementation might be a promising strategy to improve the antioxidant capacity and mitochondrial function of offspring weaned piglets by altering the intestinal microbiota.
} 


\section{Introduction}

The gastrointestinal tract (GIT) of mammalian animals has been known as a harbor of microbes [1]. The gut microbes play crucial roles in nutrients metabolism, intestinal barrier function, and immune function [2, 3]. Accumulating evidence showed that healthy maternal gut microbes are essential for the growth and health of their offspring. Microbes can be transmitted to the offspring through direct contact with the birth canal during parturition and colostrum or milk during lactation, which contributes to long-term health benefits in offspring [4-6]. However, maternal gut microbes and milk or colostrum quality are influenced by maternal diet compositions, and thus, maternal dietary intervention may be an effective way to improve offspring's overall health.

Piglets are highly susceptible to intestinal structural abnormalities and functional disorders due to their immature immune system and lack of diverse intestinal microbiota, resulting in increased incidence of diarrhea, growth retardation, and even death. Oxidative stress occurs under a condition when the production of reactive oxygen (ROS) and their elimination by the antioxidant mechanism is imbalanced. The sows undergo systematic oxidative stress during late pregnancy and lactation, which does not fully recover until weaned and could affect their offspring's health [7]. Furthermore, there is also a high correlation between maternal and fetal (cord blood) plasma antioxidant markers, suggesting that maternal oxidative stress status can transfer to the fetus [8]. Therefore, late gestation and early postnatal periods are the critical window periods for oxidative stress regulation [9].

Probiotics are defined as "live microorganisms which when administered in adequate amounts confer a health benefit on the host" [10]; prebiotics are defined as a "nonviable food component that confers a health benefit on the host associated with modulation of the microbiota"; Gibson and Roberfroid introduced the term "synbiotic" to describe a combination of synergistically acting probiotics and prebiotics $[11,12]$. Currently, increasing evidence has indicated that probiotic/synbiotic supplementation could improve antioxidant capacity and reduce oxidative stress [13]. Lactobacillus acidophilus and Lactobacillus bulgaricus, as the lactic acid bacteria, have been reported to exhibit antioxidant capacity by chelating metal ions and scavenging ROS [14]. $\mathrm{Nie}$ et al. reported that Lactobacillus frumenti improved antioxidant capacity via nitric oxide production mediated by nitric oxide synthase 1 activation in intestinal epithelial cells [15]. As a probiotic yeast, Saccharomyces cerevisiae (S. cerevisiae) showed strong antioxidant activity, reducing nitric oxide and hydroxyl radical scavenging activity [16]. Several studies have reported that the administration of probiotics or synbiotics during gestation and lactation have been considered as a potential strategy to improve the growth performance and modulate intestinal microbiota of offspring piglets [17-19]. Furthermore, maternal dietary fiber supplementation during gestation has an important role in improving the antioxidative capacity of their offspring through modulating the composition of the gut microbiota [20]. However, whether maternal probiotic or synbiotic supple- mentation during gestation and lactation can change the intestinal antioxidant capacity and mitochondrial function of offspring by altering the gut microbiota remains unclear.

Our previous study has found that dietary synbiotic supplementation to sows during pregnancy and lactation can improve piglet's survival and lipid metabolism by altering gut microbiota diversity and composition [21]. Therefore, we hypothesized that maternal probiotic or synbiotic supplementation could improve the antioxidant capacity and mitochondrial function by altering the gut microbiota of offspring weaned piglets. Considering that Bama mini-pigs' anatomy and physiology are similar to humans' [22, 23], Bama mini-pigs were chosen as a research model in this study. Our findings may, in turn, have important implications for understanding the link between maternal diets and infant intestinal health.

\section{Materials and Methods}

2.1. Experimental Design and Animal Management. The animal use and animal trials in this study have been approved by the Animal Care and Use Committee of the Institute of Subtropical Agriculture, Chinese Academy of Sciences.

A total of 64 pregnant Bama mini-sows with similar physical conditions with 3-5 parities were randomly assigned to the control group (sows fed a basal diet), antibiotic group (sows fed a basal diet supplemented with $50 \mathrm{~g} / \mathrm{t}$ virginiamycin), probiotic group (sows fed a basal diet supplemented with $200 \mathrm{~mL} / \mathrm{d}$ probiotic fermentation broth per pig), or synbiotic group (sows fed a basal diet supplemented with $200 \mathrm{~mL} / \mathrm{d}$ probiotic fermentation broth per pig and $500 \mathrm{~g} / \mathrm{t}$ xylo-oligosaccharides). From mating (day 0) to day 104 of pregnancy, the sows were individually housed in gestation crates $(2.2 \mathrm{~m} \times 0.6 \mathrm{~m})$. On day 105 , the sows were transferred to individual farrowing units $(2.2 \mathrm{~m} \times 1.8 \mathrm{~m})$.

After weaning on day 28 , two piglets per litter close to the average body weight were selected for the remaining of the trial, and four piglets from the same group were fed in one pen, and each group consisted of eight pens (replicates). Basal diets for the sows and piglets designed according to (NY/T65-2004), the Chinese nutrient requirements of swine in china (Supplementary Table 1 and Table 2) [24]. Sows were fed twice daily (at 8:00 and 17:00) according to their body conditions. Sows and piglets had available ad libitum access to water during the trial period. Hunan Lifeng Biotechnology Co., Ltd. (Changsha, China) provided the viable probiotic fermentation broth (Lactobacillus plantarum B90 (CGMCC1.12934) $\geq 1.0 \times 10^{8}$ CFU/g; S.cerevisiae P11 (CGMCC2.3854) $\geq 0.2 \times 10^{8} \mathrm{CFU} / \mathrm{g}$ ). Shandong Longlive Biotechnology Co., Ltd. (Dezhou, China) afforded the xylo-oligosaccharides (XOS), which contained the xylobiose, xylotriose, and xylotetraose $(\geq 35 \%)$.

2.2. Sampling. On day 30 of post-weaning, eight piglets (one piglet per replicate) from each group were selected and weighed at $12 \mathrm{~h}$ after the last feeding. Blood samples $(10 \mathrm{~mL})$ were collected from the precaval vein into heparintreated tubes, and the plasma was obtained by centrifuging at $3,500 \mathrm{~g}$ for $10 \mathrm{~min}$ at $4^{\circ} \mathrm{C}$ and then stored at $-20^{\circ} \mathrm{C}$ for further analysis. The piglets were then sacrificed using electrical 
stunning $(120 \mathrm{~V}, 200 \mathrm{~Hz})$, and the contents of the jejunum $(10 \mathrm{~cm}$ below the flexure of duodenum-jejunum) and colon (middle position) were collected, immediately frozen in liquid nitrogen, and stored at $-80^{\circ} \mathrm{C}$ for bacterial DNA extraction. In addition, the intestinal tissues of the jejunum and the colon were excised and rinsed with ice-cold physiological saline. The mucosa scrapings were collected, immediately frozen in liquid nitrogen, and stored at $-80^{\circ} \mathrm{C}$ for further analyses.

\subsection{Determination of Plasma and Intestinal Mucosa} Antioxidant Capacity. Approximately $100 \mathrm{mg}$ of frozen jejunum and colon tissues was removed quickly and homogenized with ice-cold physiologic saline $(1: 9, w / v)$ and then centrifuged at $2,000 \mathrm{~g}$ for $20 \mathrm{~min}$ at $4^{\circ} \mathrm{C}$. The intestinal supernatants were used for further analysis. Plasma and intestinal antioxidant indicators, including catalase (CAT), superoxide dismutase (SOD), glutathione (GSH), and glutathione peroxidase (GSH-Px), as well as malondialdehyde (MDA) were analyzed by ELISA assay kits from Jiangsu Meimian Institute (Mei mian, Yancheng, China). The total antioxidant capacity (T-AOC) and $\mathrm{H}_{2} \mathrm{O}_{2}$ assay kits were purchased from Nanjing Jiancheng Bioengineering Institute (Nanjing, China). The test for each index was carried out according to the instructions of the kits. The absorbance values were read on a Multiscan Spectrum Spectrophotometer (Tecan, Infinite M200 Pro, Switzerland). The jejunal and colonic mucous antioxidant parameters were normalized to the total protein concentration $(\mathrm{mg} / \mathrm{L})$ quantified by the Pierce BCA Protein Assay Kit (CoWin Biosciences, Suzhou, China).

\subsection{Determination of Intestinal Mucosa ATP Concentration.} The ATP concentration of the jejunal and colonic mucosa was determined using the ATP assay kit (Mei mian, Yancheng, China) based on firefly luciferase by a Multiscan Spectrum Spectrophotometer (Tecan, Infinite M200 Pro, Switzerland). The methods for intestinal tissue homogenization and total protein quantification were the same as mentioned above.

\subsection{Determination of Mitochondrial Complex I and III} Activities. The NADH ubiquinone oxidoreductase complex I and III activities in the jejunal and colonic mucosa were assessed using commercially available kits (Comin bio. Co., Suzhou, China), according to the manufacturer's instructions. The methods for intestinal tissue homogenization and total protein quantification were the same as mentioned above. The complex I and III activities were normalized to the total protein.

2.6. Real-Time Quantitative PCR Analysis of Intestinal Mucosa Antioxidant-Related Genes and MitochondrialRelated Genes. Total RNA was extracted from the frozen jejunal and colonic mucosa using a Trizol Reagent (Magen, Guangzhou, China) according to the manufacturers' protocol. The total RNA (1,000 ng) was used as a template for the cDNA reaction, which was synthesized using a PrimeScript RT reagent kit with gDNA Eraser (TaKaRa, Dalian, China). Real-time PCR analysis was performed on the LightCycler ${ }^{\circledR} 480$ II Real-Time PCR System (Roche, Basel, Swiss) (384-cell standard block). Pig-specific primers were designed and synthesized by Sangon Biotech (Shanghai, China) Co., Ltd (Table 1). The specificity of the primers was examined by the Primer-BLAST tool (https://www.ncbi.nlm.nih.gov/ tools/primer-blast) and confirmed by single peaks in the melting curves. The reaction mixture $(10 \mu \mathrm{L})$ consisted of $5.0 \mu \mathrm{L}$ SYBR Premix Ex TaqTM (AG11701; Accurate Biotechnology, Changsha, China), $2.0 \mu \mathrm{L}$ of template DNA, $0.25 \mu \mathrm{L}$ of each primer, and $2.5 \mu \mathrm{L}$ of double-distilled water. The PCR amplification conditions were followed according to the instructions of SYBR Green Premix. The relative levels of the gene expression were analyzed using $2^{-\Delta \Delta \mathrm{Ct}}$ value, and the reference gene $\beta$-actin was used as an internal control.

\subsection{Quantification of the Intestinal Mucosa Mitochondrial} DNA Content. Total DNA was extracted from the jejunal and colonic mucosa of each piglet using a DNAiso Reagent according to the manufacturer's protocol (Accurate Biotechnology, Changsha, China). The concentration of extracted DNA was measured at $260 \mathrm{~nm}$ with a NanoDrop One Microvolume UV-Vis Spectrophotometer (Thermo Scientific, Waltham, USA), and the extracted DNA was stored at $-20^{\circ} \mathrm{C}$ until further use. The content of mitochondrial DNA (mtDNA) relative to the nuclear genomic DNA was measured by amplifying the $\mathrm{mt} \mathrm{D}$-loop and nuclear-encoded $\beta$-actin gene using a real-time PCR assay as described above. The primer sequences for $\mathrm{mt} \mathrm{D}$-loop and G6PC are presented in Table 2 . The mtDNA expression $2^{-\Delta \Delta C t}$ value was calculated, and the reference gene G6PC was used as an internal control.

2.8. Real-Time Quantitative PCR Analysis for Jejunal and Colonic Microbiota Abundances. The total bacterial genomic DNA was extracted from $300 \mathrm{mg}$ of jejunal and colonic luminal contents using a Mag-Bind ${ }^{\circledR}$ Stool DNA Kit (Omega, Guangzhou, China) according to the manufacturer's protocol. The primers of the selected genes are listed in Table 3. The reaction mixture $(10 \mu \mathrm{L})$ consisted of $5.0 \mu \mathrm{L}$ SYBR Premix Ex Taq TM (AG11701; Accurate Biotechnology, Changsha, China), $2.0 \mu \mathrm{L}$ of template DNA, $0.25 \mu \mathrm{L}$ of each primer, and $2.5 \mu \mathrm{L}$ of double-distilled water. The standard curves of each gene were generated with 10 -fold serial dilutions of the respective 16S rRNA genes [25]. The qPCR amplification was carried out according to the instructions of SYBR green premix (Takara Biotechnology, Dalian, China). Melting curves were checked for each gene after amplification. The results are expressed as Lg16S ribosomal DNA gene copies/g intestine contents.

2.9. Statistical Analysis. Statistical analyses were performed using the SPSS 22.0 statistical software (SPSS Inc., Chicago, IL). Data were analyzed by one-way ANOVA, and comparative analyses were conducted using the Tukey post hoc test. Statistical results are presented as means \pm standard error of the mean (SEM). Differences were considered significant if $P<0.05$, and $0.05 \leq P<0.10$ was considered a trend. The $\mathrm{R}$ package of "Hmisc" was used for calculating Spearman's correlation coefficient. 
TAble 1: Primer sequences used for intestinal mucosa antioxidant-related genes and mitochondrial-related genes.

\begin{tabular}{|c|c|c|c|}
\hline Genes $^{\mathrm{a}}$ & Primers $\left(5^{\prime}-3^{\prime}\right)$ & Size (bp) & Accession NO. \\
\hline \multirow{2}{*}{$\beta$-Actin } & F: GATCTGGCACCACACCTTCTACAAC & \multirow{2}{*}{107} & \multirow{2}{*}{ XM_021086047.1 } \\
\hline & R: TCATCTTCTCACGGTTGGCTTTGG & & \\
\hline \multirow{2}{*}{ CAT } & F: AGCCTACGTCCTGAGTCTCTGC & \multirow{2}{*}{90} & \multirow{2}{*}{ NM_214301.2 } \\
\hline & R: TCCATATCCGTTCATGTGCCTGTG & & \\
\hline \multirow{2}{*}{ GPx1 } & F: TGCTCATTGAGAACGTAGCGT & \multirow{2}{*}{161} & \multirow{2}{*}{ NM_214201.1 } \\
\hline & R: CAGGATCTCCCCATTCTTGGC & & \\
\hline \multirow{2}{*}{ GPx4 } & F: GATTCTGGCCTTCCCTTGC & \multirow{2}{*}{173} & \multirow{2}{*}{ NM_214407.1 } \\
\hline & R: TCCCCTTGGGCTGGACTTT & & \\
\hline \multirow{2}{*}{ Keap1 } & F: CGCCTCATCGAGTTCGCTTACAC & \multirow{2}{*}{107} & \multirow{2}{*}{ NM_001114671.1 } \\
\hline & R: GCACGGACCACACTGTCAATCTG & & \\
\hline \multirow{2}{*}{ CuZnSOD } & F: CCAGTGCAGGTCCTCACTTCAATC & \multirow{2}{*}{172} & \multirow{2}{*}{ NM_001190422.1 } \\
\hline & R: CGGCCAATGATGGAATGGTCTCC & & \\
\hline \multirow{2}{*}{ MnSOD } & F: GGACAAATCTGAGCCCTAACG & \multirow{2}{*}{159} & \multirow{2}{*}{ NM_214127.2 } \\
\hline & R: CCTTGTTGAAACCGAGCC & & \\
\hline \multirow{2}{*}{ NQO1 } & F: GTGGAAGCCGCAGACCTTGTG & \multirow{2}{*}{83} & \multirow{2}{*}{ NM_001159613.1 } \\
\hline & R: CGTTCAAACCAGCCTTTCAGAATAGC & & \\
\hline \multirow{2}{*}{ Nrf1 } & F: CGATGCTTCAGAATTGCCAACTACAG & \multirow{2}{*}{125} & \multirow{2}{*}{ XM_021078993.1 } \\
\hline & R: GCGTTGTCTGGATGGTCATCTCAC & & \\
\hline \multirow{2}{*}{ Nrf2 } & F: CCAATTCAGCCAGCACAACACATC & \multirow{2}{*}{149} & \multirow{2}{*}{ XM_003133500 } \\
\hline & R: GACTGAGCCTGGTTAGGAGCAATG & & \\
\hline \multirow{2}{*}{ ATP5A1 } & F: ACGCCATTGATGGAAAGGGT & \multirow{2}{*}{98} & \multirow{2}{*}{ NM_001185142.1 } \\
\hline & R: TGGTTCCCGCACAGAGATTC & & \\
\hline ATP5B & F: CATGTTGGGCTTTGTGGGTC & 139 & XM 0019294104 \\
\hline AITJD & R: ATAGTCTCTGGCAGGCTGGA & 103 & A1V1_001929410.4 \\
\hline $\mathrm{PGC} 1 \alpha$ & F: ATGGAGCAATAAAGCGAAGAGCATTTG & 101 & NM 2139632 \\
\hline 1 Gera & R: GAGGAGGGTCATCATTTGTGGTCAG & 101 & $10101-210300.2$ \\
\hline TFAM & F: AAATTGCTGAGCTGTGGAGGGAAC & 82 & NM 0011302111 \\
\hline IFAMI & R: TACACCTGCCAGTCTGCCCTATAAG & 82 & N1V1_001150211.1 \\
\hline
\end{tabular}

${ }^{\mathrm{a} C A T}$ : catalase; GPx1: glutathione peroxidase 1; GPx4: glutathione peroxidase 4; Keap1: kelch-like ECH-associated protein 1; CuZnSOD: copper- and zinccontaining superoxide dismutase; MnSOD: manganese-containing superoxide dismutase; NQO1: nicotinamide adenine dinucleotide (phosphate) dependency quinone oxidoreductase 1; Nrf1: nuclear factor erythroid 2-related factor 1; Nrf2: nuclear factor erythroid 2-related factor 2; mtDNA: mitochondrial DNA; ATP5A1: adenosine triphosphate synthase alpha subunit; ATP5B: adenosine triphosphate synthase $\beta$; PGC-1 $\alpha$ : peroxisome proliferator-activated receptor $\gamma$ coactivator- $1 \alpha$; TFAM: mitochondrial transcription factor A.

TABle 2: Primer sequences used for intestinal mucosa mtDNA copy number analysis.

\begin{tabular}{lccc}
\hline Gene $^{\mathrm{a}}$ & Primers $\left(5^{\prime}-3^{\prime}\right)$ & Size $(\mathrm{bp})$ & Accession NO. \\
\hline mt D-loop & F: GATCGTACATAGCACATATCATGTC & 198 & AF276923 \\
& R: GGTCCTGAAGTAAGAACCAGATG & & NM_001113445.1 \\
G6PC & F: AAGCCAAGCGAAGGTGTGAGC & 165 & R: GGAACGGGAACCACTTGCTGAG \\
\hline
\end{tabular}

amt D-loop: mitochondria DNA loop; G6PC: glucose-6-phosphatase.

\section{Results}

3.1. Effect of Maternal Probiotic or Synbiotic Supplementation on Piglets' Plasma Redox Status. To explore whether maternal probiotic or synbiotic supplementation contributes to the systemic redox status, we determined the antioxidant and oxidative parameters in plasma. As shown in Figure 1, in comparison with the control group, the activities of CAT,
GSH-Px, and SOD were higher, whereas the concentrations of $\mathrm{MDA}$ and $\mathrm{H}_{2} \mathrm{O}_{2}$ were lower in the probiotic group $(P<0.05)$. Synbiotic supplementation increased $(P<0.05)$ plasma CAT, GSH-Px, and SOD activities compared with the control group. Meanwhile, plasma GSH-Px, SOD, and T-AOC activities in the probiotic and synbiotic groups were elevated $(P<0.05)$ compared with the antibiotic group. These findings suggest that maternal probiotic or synbiotic 
TABLe 3: Primer sequences used for bacteria 16S rRNA.

\begin{tabular}{|c|c|c|c|c|}
\hline Bacteria & Sequence $5^{\prime}-3^{\prime}$ & Length (bp) & Reference & Annealing temp, ${ }^{\circ} \mathrm{C}$ \\
\hline \multirow{2}{*}{ Total bacteria } & F: GTGSTGCAYGGYYGTCGTCA & \multirow{2}{*}{123} & \multirow{2}{*}[26]{} & \multirow{2}{*}{60} \\
\hline & R: ACGTCRTCCMCNCCTTCCTC & & & \\
\hline \multirow{2}{*}{ Bacteroidetes } & F: GGARCATGTGGTTTAATTCGATGAT & \multirow{2}{*}{126} & \multirow{2}{*}{ [27] } & \multirow{2}{*}{60} \\
\hline & R: AGCTGACGACAACCATGCAG & & & \\
\hline \multirow{2}{*}{ Firmicutes } & F: GGAGYATGTGGTTTAATTCGAAGCA & \multirow{2}{*}{126} & \multirow{2}{*}{ [27] } & \multirow{2}{*}{60} \\
\hline & R: AGCTGACGACAACCATGCAC & & & \\
\hline \multirow{2}{*}{ Bifidobacterium } & F: TCGCGTCYGGTGTGAAAG & \multirow{2}{*}{128} & \multirow{2}{*}{ [28] } & \multirow{2}{*}{62} \\
\hline & R: GGTGTTCTTCCCGATATCTACA & & & \\
\hline \multirow{2}{*}{ Clostridium cluster IV } & F: GCACAAGCAGTGGAGT & \multirow{2}{*}{240} & \multirow{2}{*}{ [29] } & \multirow{2}{*}{54} \\
\hline & R: CTTCCTCCGTTTTGTCAA & & & \\
\hline \multirow{2}{*}{ Escherichia coli } & F: CATGCCGCGTGTATGAAGAA & \multirow{2}{*}{95} & \multirow{2}{*}[30]{} & \multirow{2}{*}{62} \\
\hline & R: CGGGTAACGTCAATGAGCAAA & & & \\
\hline \multirow{2}{*}{ Lactobacillus } & F: AGCAGTAGGGAATCTTCCA & \multirow{2}{*}{345} & \multirow{2}{*}[31]{} & \multirow{2}{*}{62} \\
\hline & R: ATTCCACCGCTACACATG & & & \\
\hline
\end{tabular}
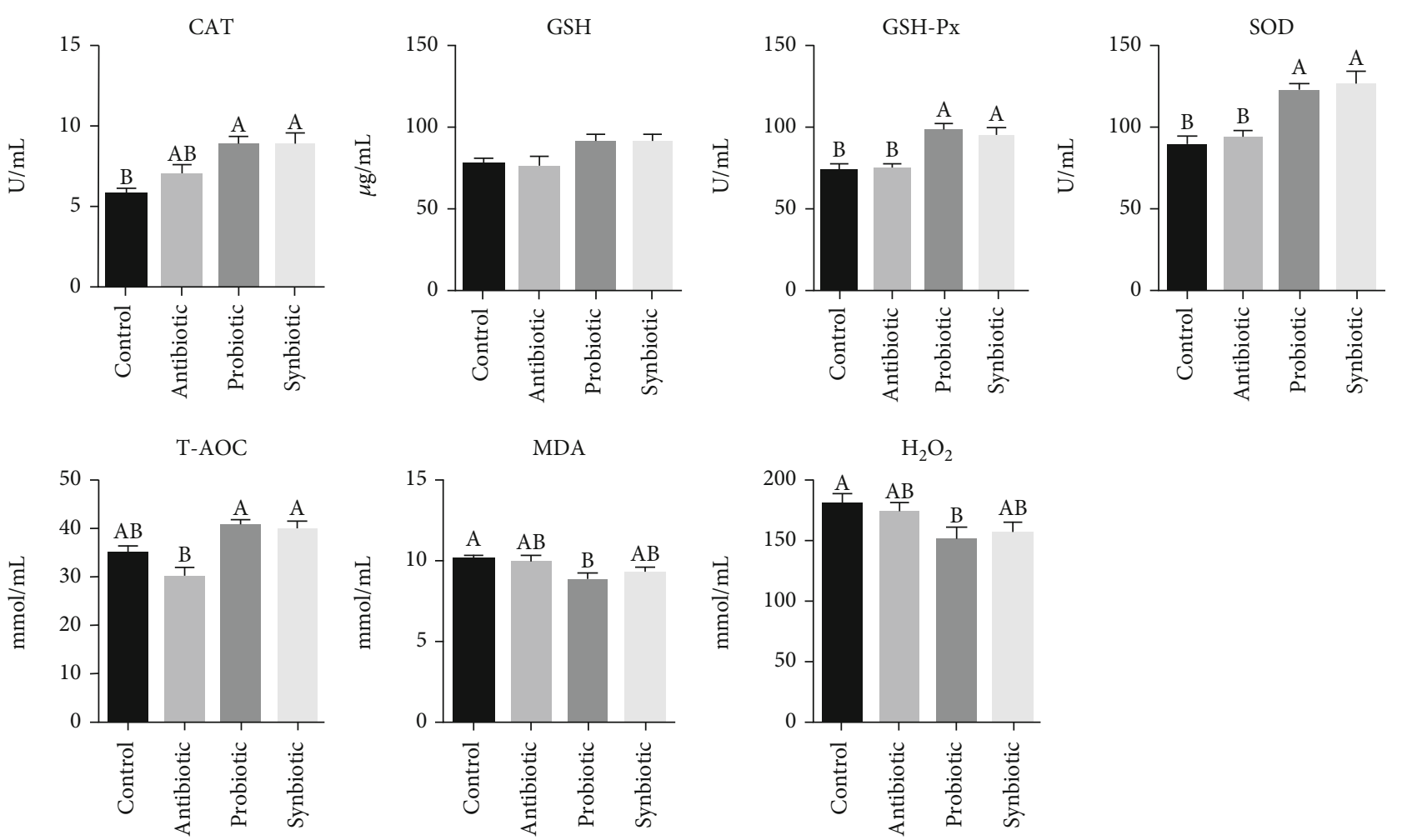

Figure 1: Effect of maternal probiotic or synbiotic supplementation during gestation and lactation on piglets' plasma redox status. Data are expressed as means $\pm \operatorname{SEM}(n=8)$. Values with different letters mean statistically significant differences $(P<0.05)$. Values with no letters mean no statistically significant differences among the groups $(P>0.05)$. CAT: catalase; GSH: glutathione; GSH-Px: glutathione peroxidase; SOD: superoxide dismutase; T-AOC: total antioxidant capacity; MDA: malondialdehyde.

supplementation could improve the systemic antioxidant capacity of offspring piglets.

\subsection{Effect of Maternal Probiotic or Synbiotic Supplementation} on Piglets' Intestinal Antioxidant Capacity. We further investigated the role of probiotic or synbiotic on jejunal and colonic antioxidant capacity. As shown in Figure 2, in the jejunum, the activities of GSH-Px and SOD were increased in the probiotic group $(P<0.05)$, and the GSH, GSH-Px, and T-AOC activities were increased in the synbiotic group $(P<0.05)$ when compared with the control group. In addition, maternal probiotic supplementation increased $(P<0.05)$ the GSH-Px and SOD activities, while maternal synbiotic supplementation increased $(P<0.05)$ the GSH-Px 

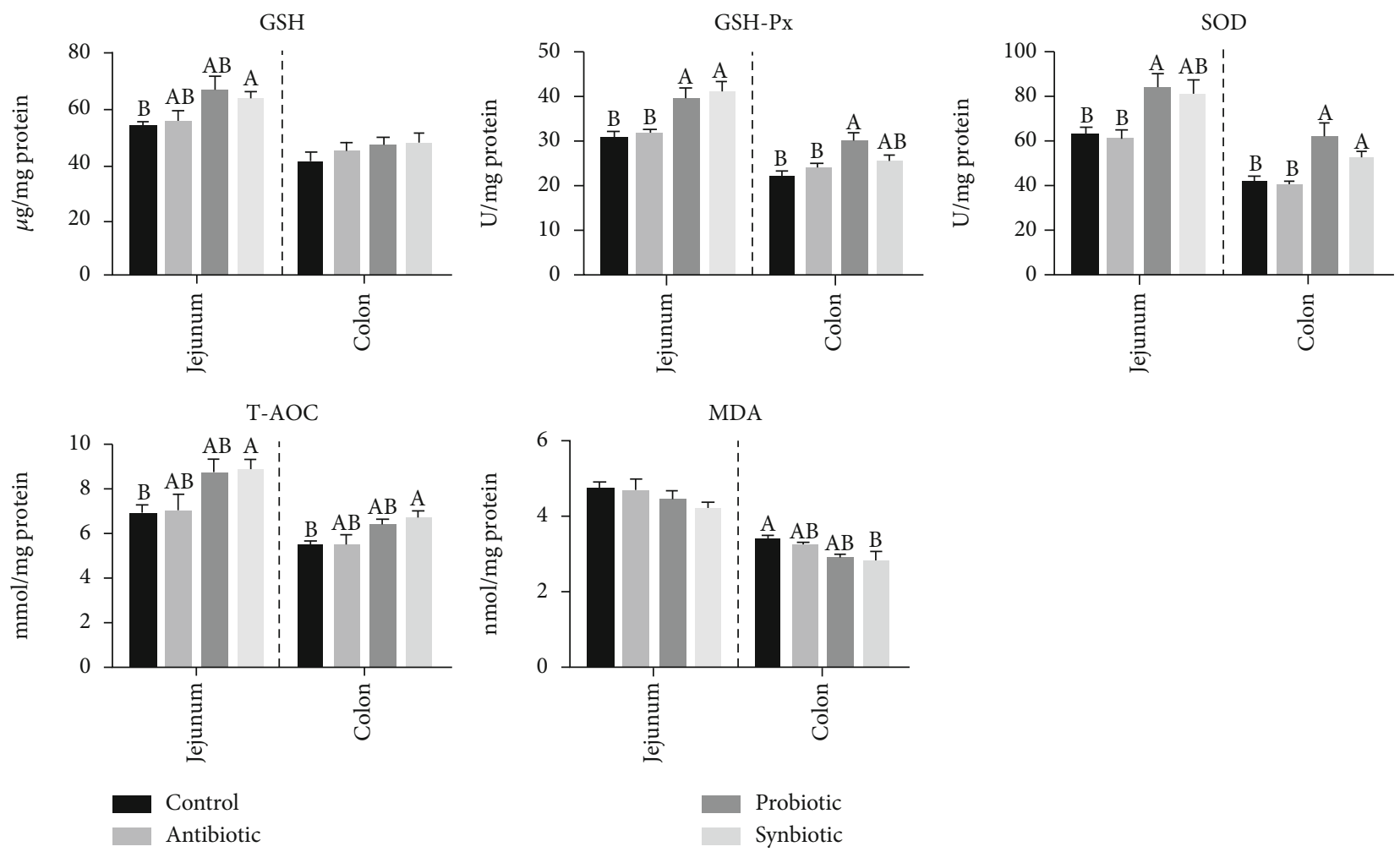

FIGURE 2: Effect of maternal probiotic or synbiotic supplementation during gestation and lactation on piglets' oxidant/antioxidant status in the jejunum and colon. Data are expressed as means \pm SEM $(n=8)$. Values with different letters mean statistically significant differences $(P<0.05)$. Values with no letters mean no statistically significant differences among the groups $(P>0.05)$. GSH: glutathione; GSH-Px: glutathione peroxidase; SOD: superoxide dismutase; T-AOC: total antioxidant capacity; MDA: malondialdehyde.

activity compared with the antibiotic group. In the colon, compared with the control group, the GSH-Px and SOD activities were higher $(P<0.05)$ in the probiotic group, as well as the SOD and T-AOC activities in the synbiotic group, while the MDA concentration was lower $(P<0.05)$ in the synbiotic group. In addition, the GSH-Px and SOD activities in the probiotic group and the SOD activity in the synbiotic group were increased $(P<0.05)$ in comparison to the antibiotic group. Therefore, these results indicate that maternal probiotic or synbiotic supplementation facilitates improving the intestinal antioxidant capacity in offspring piglets.

\subsection{Effect of Maternal Probiotic or Synbiotic Supplementation} on Piglets' Intestinal Antioxidant Enzyme-Related Genes. To investigate the molecular mechanism by which maternal probiotic or synbiotic supplementation may influence the intestinal antioxidant function, the expression of genes related to the antioxidant capacity was determined. Maternal synbiotic supplementation upregulated $(P<0.05)$ the relative mRNA expressions of CuZnSOD, Nrf1, and Nrf2 in the jejunum compared with the control and antibiotic groups. The mRNA expression of GPx1 was tended to upregulate in the probiotic $(P=0.090)$ and synbiotic $(P=0.068)$ groups compared with the control group. Interestingly, the mRNA expressions of GPx4, Nrf1, and Nrf2 in the colon were lower $(P<0.05)$ in the antibiotic group than those in the control group. Meanwhile, the mRNA expression of colonic Nrf2 in the probiotic
$(P=0.095)$ and synbiotic $(P=0.087)$ groups was tended to increase compared with the antibiotic group (Table 4). Our data indicated that maternal probiotic or synbiotic supplementation could improve the jejunal antioxidant enzymerelated genes to some extent, while had no significant effect on the colon.

\subsection{Effect of Maternal Probiotic or Synbiotic Supplementation} on Piglets' Intestinal ATP Concentrations, Mitochondrial Complex I and III Activities. As enterocytes have high energy demands to maintain renewal and the transport of nutrients, the effect of maternal probiotic or synbiotic supplementation on the mitochondrial oxidative metabolism and the jejunal and colonic mucosal ATP production were measured. Although there were no significant differences in the jejunal ATP concentration among the four groups, the concentration of jejunal ATP was higher by $9.1 \%$ and $18.7 \%$ in the probiotic and synbiotic groups, respectively, than the control group (Figure S1). The jejunal mitochondrial complex I activity in the antibiotic group was higher compared with the other three groups. Compared to the control group, the activity of colonic mitochondrial complex I was higher by $20.16 \%$ and $44.82 \%$ in the antibiotic and probiotic groups, while tended to decreased $(P=0.086)$ in the synbiotic group (Figure S2A). However, there were no significant differences in the mitochondrial complex III activity in the jejunum and colon among the four groups (Figure S2B). 
TABLE 4: Effect of maternal probiotic or synbiotic supplementation during gestation and lactation on piglets' antioxidant-related gene expressions in the jejunum and colon.

\begin{tabular}{|c|c|c|c|c|c|c|}
\hline \multirow{2}{*}{ Items $^{\mathrm{a}}$} & \multicolumn{4}{|c|}{ Dietary treatment } & \multirow{2}{*}{ SEM } & \multirow{2}{*}{$P$ values } \\
\hline & Control & Antibiotic & Probiotic & Synbiotic & & \\
\hline \multicolumn{7}{|l|}{ Jejunum } \\
\hline CAT & 1.12 & 0.89 & 1.66 & 1.61 & 0.143 & 0.181 \\
\hline GPx1 & 1.05 & 1.38 & 1.62 & 1.67 & 0.093 & 0.049 \\
\hline GPx4 & 1.11 & 1.26 & 1.49 & 1.35 & 0.090 & 0.482 \\
\hline Keap1 & 1.02 & 0.93 & 1.09 & 1.12 & 0.036 & 0.305 \\
\hline CuZnSOD & $1.09^{\mathrm{b}}$ & $1.01^{\mathrm{b}}$ & $1.26^{\mathrm{ab}}$ & $1.82^{\mathrm{a}}$ & 0.102 & 0.020 \\
\hline MnSOD & 1.05 & 1.22 & 1.25 & 1.52 & 0.077 & 0.188 \\
\hline NQO1 & 1.18 & 1.49 & 1.18 & 1.29 & 0.142 & 0.875 \\
\hline Nrf1 & $1.02^{\mathrm{b}}$ & $0.95^{\mathrm{b}}$ & $1.06^{\mathrm{ab}}$ & $1.31^{\mathrm{a}}$ & 0.038 & 0.002 \\
\hline Nrf2 & $1.11^{\mathrm{b}}$ & $1.12^{\mathrm{b}}$ & $1.33^{\mathrm{ab}}$ & $1.63^{\mathrm{a}}$ & 0.072 & 0.032 \\
\hline \multicolumn{7}{|l|}{ Colon } \\
\hline CAT & 1.05 & 0.74 & 0.82 & 0.78 & 0.049 & 0.076 \\
\hline GPx1 & 1.03 & 0.71 & 0.90 & 1.00 & 0.053 & 0.146 \\
\hline GPx4 & $1.08^{\mathrm{a}}$ & $0.38^{\mathrm{b}}$ & $0.90^{\mathrm{ab}}$ & $0.95^{\mathrm{ab}}$ & 0.087 & 0.020 \\
\hline Keap1 & 1.00 & 0.96 & 1.04 & 0.94 & 0.028 & 0.618 \\
\hline CuZnSOD & 0.95 & 0.57 & 0.86 & 0.88 & 0.059 & 0.117 \\
\hline MnSOD & 1.06 & 0.95 & 1.25 & 1.13 & 0.070 & 0.516 \\
\hline NQO1 & 1.15 & 0.64 & 0.87 & 1.20 & 0.109 & 0.269 \\
\hline Nrf1 & $1.02^{\mathrm{a}}$ & $0.77^{\mathrm{b}}$ & $0.93^{\mathrm{ab}}$ & $1.03^{\mathrm{a}}$ & 0.033 & 0.018 \\
\hline Nrf2 & $1.07^{\mathrm{a}}$ & $0.64^{\mathrm{b}}$ & $0.75^{\mathrm{ab}}$ & $0.73^{\mathrm{ab}}$ & 0.056 & 0.014 \\
\hline
\end{tabular}

Data are expressed as means with SEM $(n=8)$. Means with different superscript letters in the same row were significantly different $(P<0.05)$. Values with no letters mean no statistically significant differences among the groups $(P>0.05) .{ }^{\mathrm{a} C A T}$ : catalase; GPx1: glutathione peroxidase 1; GPx4: glutathione peroxidase 4; Keap1: kelch-like ECH-associated protein 1; CuZnSOD: copper- and zinc-containing superoxide dismutase; MnSOD: manganese-containing superoxide dismutase; NQO1: nicotinamide adenine dinucleotide (phosphate) dependency quinone oxidoreductase 1; Nrf1: nuclear factor erythroid 2-related factor 1; Nrf2: nuclear factor erythroid 2-related factor 2.

These findings suggest that maternal probiotic or synbiotic supplementation tends to improve jejunal mitochondrial function.

\subsection{Effect of Maternal Probiotic or Synbiotic Supplementation} on Piglets' Mitochondrial Biogenesis-Related Genes in the Jejunum and Colon. Subsequently, mitochondrial biogenesisrelated genes in the intestinal mucosa were measured to understand further protective effects of maternal probiotic or synbiotic on mitochondria. As shown in Table 5, in the jejunal mucosa, maternal synbiotic supplementation increased $(P<0.05)$ the mRNA expressions of ATP5A1, ATP5B, and TFAM compared to the control group, as well as the mRNA expressions of ATP5A1 and ATP5B compared with the antibiotic group. In comparison with the probiotic group, maternal synbiotic supplementation increased $(P<0.05)$ the mRNA expression of ATP5B in piglets' jejunum. However, in the colonic mucosa, the PGC1 $\alpha$ mRNA expression level was decreased $(P<0.05)$ in the synbiotic group compared with the control group. The results demonstrated that maternal synbiotic supplementation could improve the jejunal mitochondrial biogenesis-related genes.

3.6. Effect of Maternal Probiotic or Synbiotic Supplementation on Piglets' Microbial Abundances in the Jejunum and Colon. We further investigated the effects of maternal probiotic or synbiotic supplementation on the selected intestinal microbiota abundances in offspring piglets. As shown in Figure 3, in the jejunum, maternal probiotic supplementation increased $(P<0.05)$ the relative abundances of Bacteroidetes and Bifidobacterium, and synbiotic supplementation increased $(P<0.05)$ the relative abundances of Firmicutes, Bacteroidetes, Bifidobacterium, and Lactobacillus when compared with the control group. Similarly, maternal antibiotic supplementation also increased the relative abundances of total bacteria $(P=0.066)$, Bifidobacterium $(P<0.05)$, and Lactobacillus $(P<0.05)$ compared with the control group. In the colon, the relative abundance of Bifidobacterium in the probiotic group was higher $(P<0.05)$ compared with the control and antibiotic groups. However, there were no significant differences in the abundances of total bacteria, Bacteroides, Clostridium cluster IV, and Lactobacillus among the four groups. These results indicated that maternal probiotic or synbiotic significantly changed particular bacteria abundances in offspring piglets, with an increase in the counts of beneficial bacteria in the jejunum.

3.7. Correlation Analysis of Antioxidant Index, Mitochondrial Index, and Intestinal Bacteria Abundance in the Jejunum and Colon. The correlation analysis of all measured bacterial abundance, antioxidant index, and mitochondrial function parameters is shown in Figure 4. The correlation analysis 
TABLE 5: Effect of maternal probiotic or synbiotic supplementation during gestation and lactation on mitochondrial-related gene expression in the jejunum and colon of piglets.

\begin{tabular}{|c|c|c|c|c|c|c|}
\hline \multirow{2}{*}{ Items $^{\mathrm{a}}$} & \multicolumn{4}{|c|}{ Dietary treatment } & \multirow{2}{*}{ SEM } & \multirow{2}{*}{$P$ values } \\
\hline & Control & Antibiotic & Probiotic & Synbiotic & & \\
\hline \multicolumn{7}{|l|}{ Jejunum } \\
\hline mtDNA & 1.02 & 0.997 & 1.08 & 0.936 & 0.0381 & 0.661 \\
\hline ATP5A1 & $1.02^{\mathrm{b}}$ & $0.95^{\mathrm{b}}$ & $1.17^{\mathrm{ab}}$ & $1.43^{\mathrm{a}}$ & 0.057 & 0.014 \\
\hline ATP5B & $1.03^{\mathrm{b}}$ & $1.07^{\mathrm{b}}$ & $1.20^{\mathrm{b}}$ & $1.64^{\mathrm{a}}$ & 0.066 & 0.001 \\
\hline PGC1 $\alpha$ & 1.04 & 0.80 & 1.18 & 1.14 & 0.058 & 0.110 \\
\hline TFAM & $1.05^{\mathrm{b}}$ & $1.21^{\mathrm{ab}}$ & $1.28^{\mathrm{ab}}$ & $1.51^{\mathrm{a}}$ & 0.055 & 0.016 \\
\hline \multicolumn{7}{|l|}{ Colon } \\
\hline mtDNA & 1.06 & 1.09 & 0.98 & 0.80 & 0.051 & 0.192 \\
\hline ATP5A1 & 1.03 & 0.75 & 0.89 & 0.98 & 0.042 & 0.100 \\
\hline ATP5B & 1.02 & 0.93 & 1.08 & 1.06 & 0.041 & 0.629 \\
\hline $\mathrm{PGC} 1 \alpha$ & $1.11^{\mathrm{a}}$ & $0.65^{\mathrm{ab}}$ & $1.16^{\mathrm{ab}}$ & $0.60^{\mathrm{b}}$ & 0.076 & 0.008 \\
\hline TFAM & $1.03^{\mathrm{a}}$ & $0.71^{\mathrm{b}}$ & $0.89^{\mathrm{ab}}$ & $0.86^{\mathrm{ab}}$ & 0.047 & 0.106 \\
\hline
\end{tabular}

Data are expressed as means with their SEM $(n=8)$. Values with different letters in the same row were significantly different $(P<0.05)$. Values with no letters mean no statistically significant differences among the groups $(P>0.05) .{ }^{a}$ mtDNA: mitochondrial DNA; ATP5A1: adenosine triphosphate synthase alpha subunit; ATP5B: adenosine triphosphate synthase $\beta$, polypeptide; PGC- $1 \alpha$ : peroxisome proliferator-activated receptor $\gamma$ coactivator- $1 \alpha$; TFAM: mitochondrial transcription factor A.

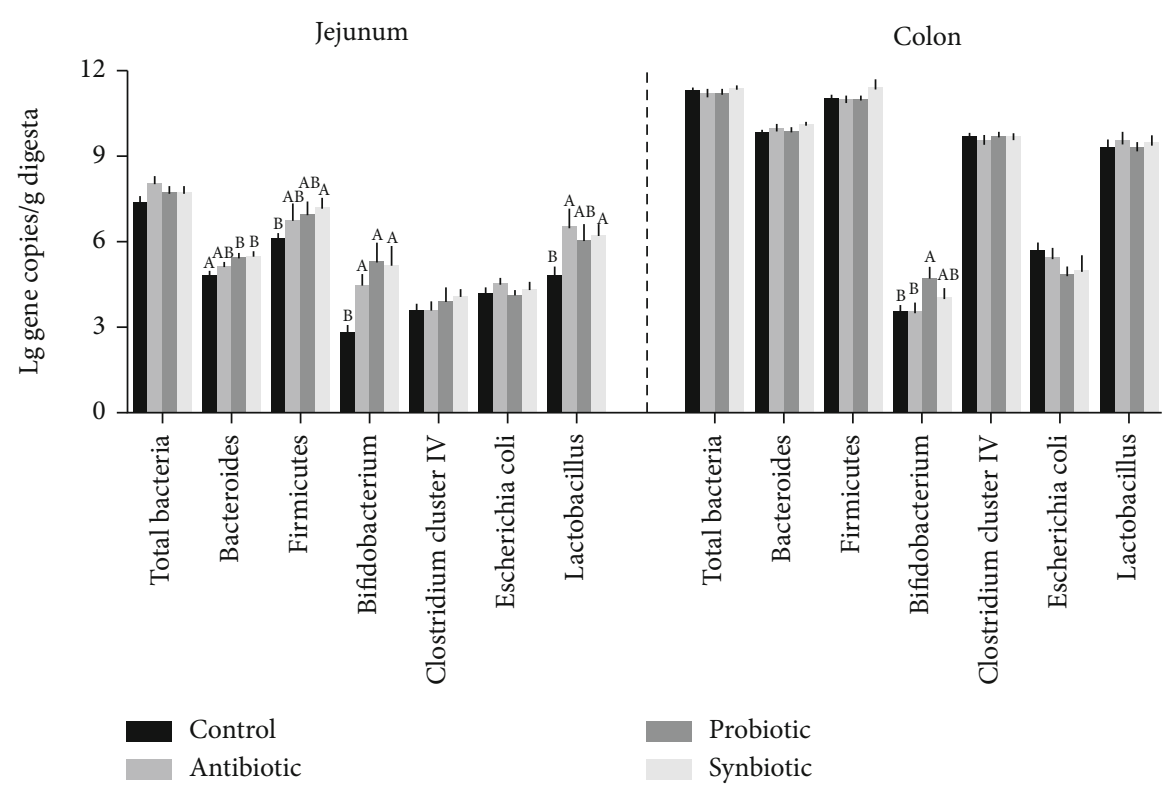

FIGURE 3: Effect of maternal probiotic or synbiotic supplementation during gestation and lactation on the copy numbers (Lg (copies/g)) of bacterial abundance in offspring piglets. Data are expressed as means \pm SEM $(n=8)$. Values with different letters mean statistically significant differences $(P<0.05)$. Values with no letters mean no statistically significant differences among the groups $(P>0.05)$.

revealed that jejunal Bifidobacterium abundance was positively correlated $(P<0.05)$ with the jejunal CAT expression and colonic GSH-Px activity but negatively correlated $(P<0.05)$ with the colonic MDA concentration. The jejunal Escherichia coli abundance was positively correlated $(P<0.05)$ with jejunal MDA concentration, while negatively correlated $(P<0.05)$ with the jejunal MnSOD expression and colonic mtDNA expression. The jejunal Lactobacillus abundance was positively correlated $(P<0.05)$ with the jejunal CAT and NQO1 expression. The jejunal Bacteroidetes was positively correlated $(P<0.05)$ with plasma CAT and GSH-Px activities, jejunal T-AOC activity and mtDNA expression, and colonic T-AOC activity. The jejunal Firmicutes abundance was positively correlated $(P<0.05)$ with colonic GSH-Px activity and jejunal CAT and NQO1 expression. The jejunal GSH-Px activity showed positive correlations $(P<0.05)$ with plasma CAT, GSH-Px, SOD, and T-AOC activities; colonic T-AOC activity; and jejunal GPx1, GPx4, Nrf1, ATP5A1, ATP5B, and TFAM mRNA levels, while showed negative correlations $(P<0.05)$ with $\mathrm{MDA}$ concentration in 


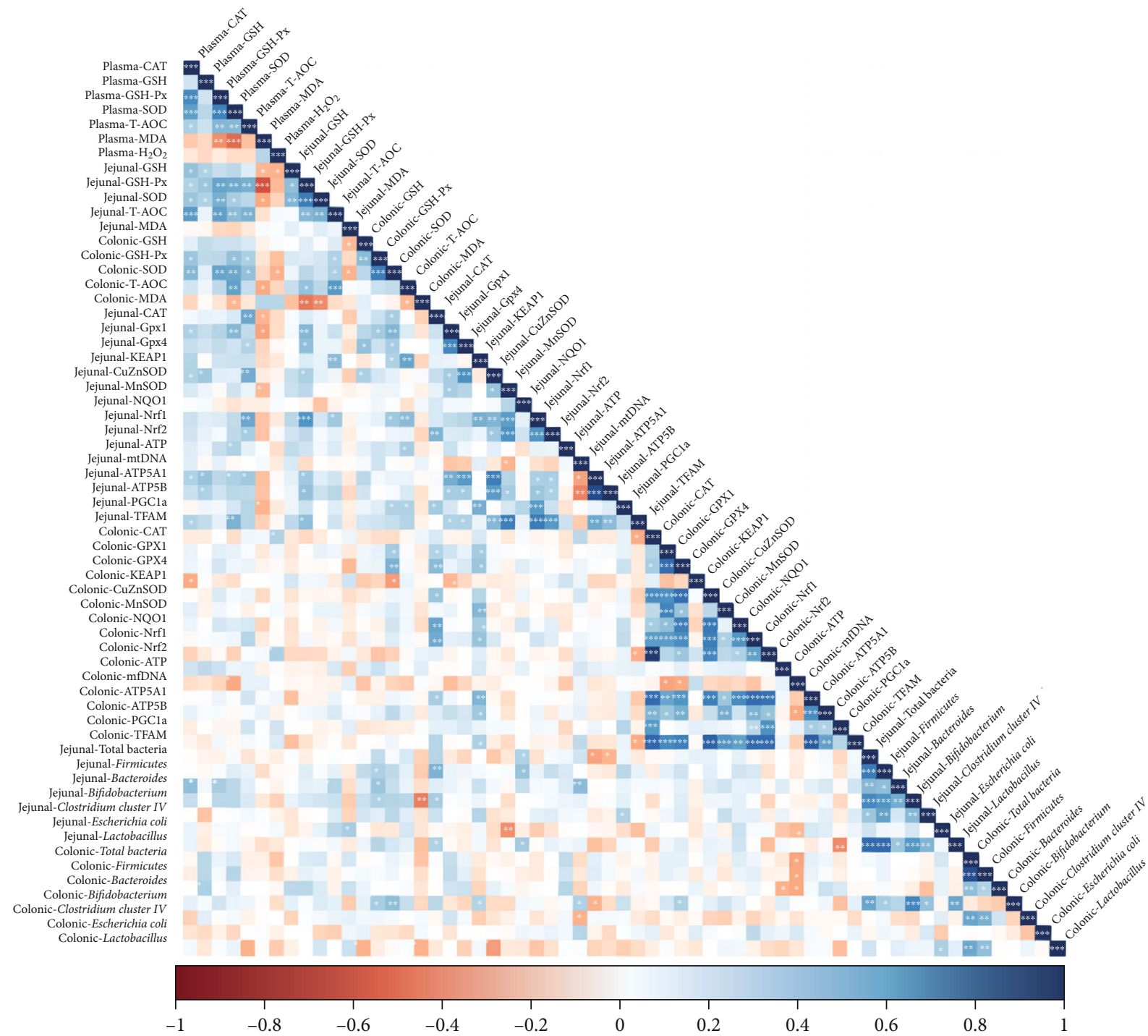

FIgURE 4: Correlation of antioxidant indexes, mitochondrial function indexes, and bacteria abundances. The R package of "corroplot" was used for generating the heat maps. The blue color represents a significant positive correlation, and red color represents a significant negative correlation. Asterisks indicate statistically significant difference: ${ }^{*} P<0.05 ;{ }^{* *} P<0.01$; ${ }^{* * *} P<0.001$. CAT: catalase; GSH: glutathione; GSH-Px: glutathione peroxidase; SOD: superoxide dismutase; T-AOC: total antioxidant capacity; MDA: malondialdehyde; GPx1: glutathione peroxidase 1; GPx4: glutathione peroxidase 4; Keap1: kelch-like ECH-associated protein 1; CuZnSOD: copper- and zinc-containing superoxide dismutase; MnSOD: manganese-containing superoxide dismutase; NQO1: nicotinamide adenine dinucleotide (phosphate) dependency quinone oxidoreductase 1; Nrf1: nuclear factor erythroid 2-related factor 1; Nrf2: nuclear factor erythroid 2-related factor 2; mtDNA: mitochondrial DNA; ATP5A1: adenosine triphosphate synthase alpha subunit; ATP5B: adenosine triphosphate synthase $\beta$, polypeptide; PGC-1 $\alpha$ : peroxisome proliferator-activated receptor $\gamma$ coactivator- $1 \alpha$; TFAM: mitochondrial transcription factor A.

the plasma and colon. The jejunal T-AOC activity showed positive correlations $(P<0.05)$ with plasma CAT, GSH-Px, SOD, and T-AOC activities; colonic GSH-Px and SOD activities; and jejunal Keap1 and Nrf1 expressions. In the colon, Bifidobacterium abundance was positively correlated $(P<0.05)$ with colonic GSH-Px and SOD activities, and GPx4 expression. Besides, colonic Lactobacillus abundance was negatively correlated with colonic GSH activity and MDA concentration.

\section{Discussion}

In humans, maternal nutritional strategies during gestation and lactation have been investigated due to their potential impact on fetal growth and development, as well as the beneficial effects on offspring's health [32]. Moreover, maternal adverse nutritional conditions may alter the structure and function of particular organs of offspring and lead to many complications later in life [33]. Previous studies have found that the administration of probiotics and prebiotics during gestation and lactation is a possible dietary strategy to benefit infant health [34]. Probiotics present many beneficial effects, and strain-specific probiotics can exhibit antioxidant activity and reduce the intestinal damage caused by oxidation [35]. The present study investigates whether dietary probiotic or synbiotic supplementation to sows during gestation and lactation affects the antioxidant capacity and mitochondrial 
function in offspring piglets and further explored whether it is associated with intestinal bacteria. We found that maternal probiotic or synbiotic supplementation during gestation and lactation significantly enhanced systemic and intestinal antioxidant capacity, improved mitochondrial biogenesis, and altered the jejunal and colonic bacteria communities in offspring piglets. Furthermore, correlation analysis revealed that jejunal and colonic microbiota abundances were significantly correlated with antioxidant enzyme activities and mitochondrial biogenesis-related indexes.

The antioxidant capacity in plasma reflects the host's systemic capacity to respond to oxidative damage. $\mathrm{H}_{2} \mathrm{O}_{2}$, as a major type of ROS, is involved in lipoperoxidation [36]. The MDA is a decomposition product of lipoperoxidation, is the important marker of oxidative stress [37]. By lowering the MDA level in plasma, it is possible to lower the degree of lipid destruction and enhance the ability of ROS scavenging. In the present study, dietary probiotic supplementation to sows decreased plasma MDA and $\mathrm{H}_{2} \mathrm{O}_{2}$ concentrations of offspring piglets. The major GSH-dependent enzymatic antioxidants are SOD, GSH-Px, and CAT, which play a vital role in scavenging ROS [38]. The GSH serves as the major endogenous antioxidant, acting as a free radical scavenger in the cell. GSH-px is a key enzyme to catalyze GSH into GSSG. Our results showed that the activity of GSH-Px in the plasma of probiotic- or synbiotic-treated piglets was significantly higher than those in the control group. However, the GSH content in the plasma of probiotic- or synbiotic-treated piglets was increased numerically but not significantly. Based on these results, we speculated that maternal probiotic or synbiotic supplementation may contribute slightly to regulate the oxidation and reduction reactions of GSH; however, the exact mechanism remained unknown. The redox status of GSH can be expressed by its half-cell redox potential (GSH/GSSG Eh) [39]. Further studies are needed to explore the effect of maternal probiotic or synbiotic supplementation on the GSSG and GSH/GSSG Eh levels to fully understand the GSH redox status.

The intestinal mucosa, the front-line barrier to food antigens, pathogens, and commensal organisms, plays a crucial role in sustaining intestinal epithelial homeostasis [40]. However, many stimuli factors (i.e., infection and inflammation) can induce the overproduction of proinflammatory cytokines and ROS to damage intestinal barrier function [41]. In the present study, consistent with the findings in plasma, the MDA content in the colonic mucosa of maternal synbiotic-treated piglets was significantly decreased. Furthermore, maternal probiotic or synbiotic supplementation can partially increase the antioxidant enzyme activities in the jejunum and colon of offspring piglets. Gu et al. reported that isomalto-oligosaccharide and Bacillus supplementation to sows during late gestation could improve the placental antioxidant capacity and the piglets' birth weight [42]. In a human study, it was found that plasma and erythrocyte MDA levels were significantly higher, and the erythrocyte GSH level was lower in the pregnant women than in the nonpregnant women, and there was a significant positive correlation in MDA, GSH-R, and GSH-P levels between maternal and cord blood erythrocyte. These results indicated that the fetus may affect by the oxidant status of pregnant women [8]. Synbiotic supplementation could improve the T-AOC level and slightly reduce MDA level in human breastmilk [43]. However, the effect of dietary probiotic or synbiotic supplementation on the antioxidant enzymes in sows' breastmilk or placenta is unclear. Further studies are necessary to explore the effect of probiotic or synbiotic on the transmission of antioxidant capacity from sows to their offspring.

The Nrf2 pathway plays an important role in the regulation of intracellular redox status [44]. We further confirmed that the relative expression of Nrf2 mRNA was increased in the jejunum of the synbiotic group. In addition, dietary synbiotic treatment upregulated the Nrf2-regulated genes, including CuZnSOD (SOD1) and GPx1. These findings indicated that maternal synbiotic supplementation could enhance the antioxidant capacity of offspring piglets by upregulating the Nrf2 pathway. The ATP production and utilization are very active in the intestinal epithelial cells [45], due to the intestinal epithelial cells maintain cell turnover every 3-5 days [46]. Therefore, energy deficits can easily lead to impaired intestinal barrier function. Mitochondria is one of the key sources of oxidative stress, as it utilizes oxygen for cellular ATP production. Oxidative stress in cells can lead to impaired mitochondrial function, including the content of mtDNA and the expression of mitochondrial genes [47]. In the present study, the jejunal ATP concentration in piglets tended to increase after the addition of probiotic or synbiotic in the sows' diets. Furthermore, maternal synbiotic supplementation significantly upregulated the mRNA expression of jejunal ATP5A1 and ATP5B, which might be responsible for the increased ATP level in the synbiotic-treated piglets. An increase in TFAM expression was observed in the jejunal mucosa of maternal synbiotic-treated piglets. It has been reported that TFAM plays a critical role in mitochondrial biogenesis and regulates the mtDNA copy number [48]. Collectively, these findings indicated that maternal probiotic or synbiotic supplementation might improve the processes of jejunum energy metabolism in offspring piglets. Moreover, in the present study, antibiotic and probiotic treatment increased the activity of colonic mitochondrial complex I, which could result in a boost of mitochondrial energy generation. Similar findings were observed in cardiac mitochondrial dysfunction of insulin-resistant rats treated with prebiotics, probiotics, and synbiotics which showed improved cardiac mitochondrial function and reduced oxidative stress [49].

Intestinal microbiota plays a crucial role in maintaining health and regulating pathogenesis in the host. Probiotics can stimulate the colonization of the piglet's gut with beneficial bacteria, enriches the gut microbiota diversity, and prevents the intestinal infection of neonatal piglets [50]. Moreover, probiotics could also exert antioxidant effects by modulating the composition of intestinal microbiota [35]. In humans, supplementing probiotic to the pregnant mother during late pregnancy could promote the colonization of the infant's gut with beneficial bacteria such as $L$. rhamnosus $G G$ or Bifidobacteria [51,52]. Our study in pigs showed that maternal probiotic supplementation significantly increased 


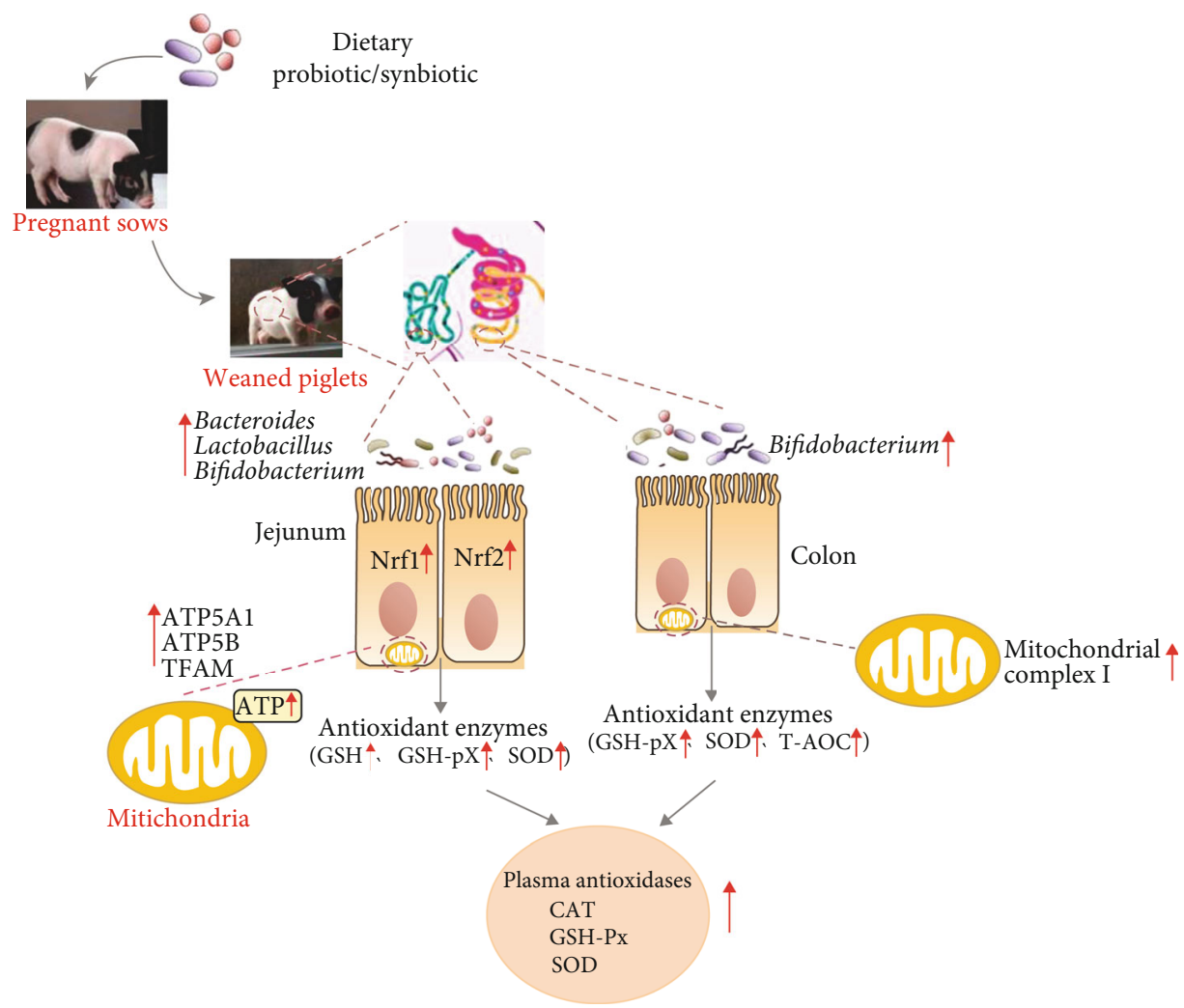

FIGURE 5: Schematic demonstrated the hypothesized mechanism of maternal probiotic or synbiotic effects on antioxidant capacity of offspring piglets. CAT: catalase; GSH: glutathione; GSH-Px: glutathione peroxidase; SOD: superoxide dismutase; T-AOC: total antioxidant capacity; Nrf1: nuclear factor erythroid 2-related factor 1; Nrf2: nuclear factor erythroid 2-related factor 2; ATP5A1: adenosine triphosphate synthase alpha subunit; ATP5B: adenosine triphosphate synthase $\beta$, polypeptide; TFAM: mitochondrial transcription factor A.

the Bacteroidetes and Bifidobacterium abundances in the jejunum and Bifidobacterium in the colon of offspring piglets. Bacteroidetes are known as short-chain fatty acids producing bacteria, which protects the mocusa of the host from damage by pathogens, provides nutrition for colonic epithelial cells, and reduces inflammation. In addition, Bacteroidetes can supply energy to the host via carbohydrate degradation and associated with the immune response of the host [53]. The present study also found that maternal synbiotic supplementation during gestation and lactation increased the relative abundances of Firmicutes, Bacteroidetes, Bifidobacterium, and Lactobacillus in the jejunum of piglets. Firmicutes were found associated with the degradation of different carbon sources, oligosaccharides, proteins, and amino acids and their fermentative metabolisms to provide energy to the host [54]. Furthermore, Lactobacillus and Bifidobacterium, as the most common probiotic species, could inhibit the growth of pathogenic bacteria to maintain the balance of the intestinal microbiota and thus alleviate the intestinal oxidative stress $[55,56]$. Previous studies research also reported that changes in the intestinal microbiota were strongly associated with oxidative stress in high-fat diet-fed mice [57]. Consistent with these findings, the correlation analysis in the present study showed that the antioxidant enzyme activities exhibited a positive correlation with the abundances of Firmicutes, Bacteroidetes, Bifidobacterium, and Lactobacillus while MDA level was negatively correlated with the abundances of jejunal
Bifidobacterium and colonic Lactobacillus. Collectively, these findings indicated that maternal probiotic or synbiotic supplementation alleviated the intestinal oxidative stress in offspring piglets, which might be attributed to the increase in the abundances of beneficial bacteria, such as Bifidobacterium.

\section{Conclusions}

The present study showed a beneficial effect of maternal probiotic (Lactobacillus Plantarum and Saccharomyces cerevisiae) or synbiotic (probiotic + xylo-oligosaccharides) supplementation during gestation and lactation on antioxidant capacity and mitochondrial biogenesis, which may partly be attributed to altered intestinal microbiota in offspring weaned piglets. However, maternal synbiotic supplementation did not show any enhanced effects compared to probiotic in offspring weaned piglets (Figure 5). These findings implicated that probiotic or synbiotic would be potential maternal dietary additives to improve offspring's intestinal health.

\section{Data Availability}

The data used to support the findings of this study are included in the article and the supplementary information files. 


\section{Conflicts of Interest}

The authors declare that there is no conflict of interest.

\section{Authors' Contributions}

KXF, YYL, and HQH designed the experiment. WK, AMAK, ZQ, XL, ZYZ, and HZL carried out the animal experience, sample collection, and sample analysis. WK performed the statistical analyses. WK, HQH, and KXF wrote the manuscript. All authors read and approved the final manuscript.

\section{Acknowledgments}

The present work was jointly supported by the National Key Research and Development Project (2018YFD0500404-4), the Special Funds for Construction of Innovative Provinces in Hunan Province (2019RS3022), the Talent Projects of Guangxi Science and Technology Department (AD17195043), and the Basic Research Program of Shenzhen Municipal Government (JCYJ20180305125139107 and GRCK2017081809101684).

\section{Supplementary Materials}

Table S1: composition and nutrient levels of the sows' basal diets (air-dry basis; \%). Table S2: composition and nutrient levels of the basal diet for weaned piglets. Figure S1: effect of maternal probiotic or synbiotic supplementation during gestation and lactation on ATP concentrations in the jejunum and colon of piglets. Figure S2: effect of maternal probiotic or synbiotic supplementation during gestation and lactation on mitochondrial complex I and III activities in the jejunum and colon of piglets. (Supplementary materials)

\section{References}

[1] M. G. Rooks and W. S. Garrett, "Gut microbiota, metabolites and host immunity," Nature Reviews. Immunology, vol. 16, no. 6, pp. 341-352, 2016.

[2] T. C. Fung, C. A. Olson, and E. Y. Hsiao, "Interactions between the microbiota, immune and nervous systems in health and disease," Nature Neuroscience, vol. 20, no. 2, pp. 145-155, 2017.

[3] Y. Belkaid and O. J. Harrison, "Homeostatic immunity and the microbiota," Immunity, vol. 46, no. 4, pp. 562-576, 2017.

[4] S. Wang, C. A. Ryan, P. Boyaval, E. M. Dempsey, R. P. Ross, and C. Stanton, "Maternal vertical transmission affecting early-life microbiota development," Trends in Microbiology, vol. 28, no. 1, pp. 28-45, 2020.

[5] Q. Shang, H. Liu, S. Liu, T. He, and X. Piao, "Effects of dietary fiber sources during late gestation and lactation on sow performance, milk quality, and intestinal health in piglets1," Journal of Animal Science, vol. 97, no. 12, pp. 4922-4933, 2019.

[6] M. Gomez de Aguero, S. C. Ganal-Vonarburg, T. Fuhrer et al., "The maternal microbiota drives early postnatal innate immune development," Science, vol. 351, no. 6279, pp. 12961302, 2016.

[7] C. B. Berchieri-Ronchi, S. W. Kim, Y. Zhao, C. R. Correa, K. J. Yeum, and A. L. Ferreira, "Oxidative stress status of highly prolific sows during gestation and lactation," Animal, vol. 5, no. 11, pp. 1774-1779, 2011.
[8] S. Arikan, D. Konukoğlu, C. Arikan, T. Akçay, and I. Davas, "Lipid peroxidation and antioxidant status in maternal and cord blood," Gynecologic and Obstetric Investigation, vol. 51, no. 3, pp. 145-149, 2001.

[9] Z. C. Luo, W. D. Fraser, P. Julien et al., "Tracing the origins of "fetal origins" of adult diseases: programming by oxidative stress?," Medical Hypotheses, vol. 66, no. 1, pp. 38-44, 2006.

[10] N. T. Williams, "Probiotics," American Journal of HealthSystem Pharmacy, vol. 67, no. 6, pp. 449-458, 2010.

[11] K. R. Pandey, S. R. Naik, and B. V. Vakil, "Probiotics, prebiotics and synbiotics- a review," Journal of Food Science and Technology, vol. 52, no. 12, pp. 7577-7587, 2015.

[12] G. R. Gibson and M. B. Roberfroid, "Dietary modulation of the human colonic microbiota: introducing the concept of prebiotics," The Journal of Nutrition, vol. 125, no. 6, pp. 1401-1412, 1995.

[13] B. Pourrajab, S. Fatahi, M. H. Sohouli, M. A. Gaman, and F. Shidfar, "The effects of probiotic/synbiotic supplementation compared to placebo on biomarkers of oxidative stress in adults: a systematic review and meta-analysis of randomized controlled trials," Critical Reviews in Food Science and Nutrition, vol. 12, no. 1, pp. 1-18, 2020.

[14] M. Y. Lin and C. L. Yen, "Antioxidative ability of lactic acid bacteria," Journal of Agricultural and Food Chemistry, vol. 47, no. 4, pp. 1460-1466, 1999.

[15] Y. Nie, J. Hu, Q. Hou et al., "Lactobacillus frumenti improves antioxidant capacity via nitric oxide synthase 1 in intestinal epithelial cells," The FASEB Journal, vol. 33, no. 10, pp. 10705-10716, 2019.

[16] M. Fakruddin, M. N. Hossain, and M. M. Ahmed, "Antimicrobial and antioxidant activities of Saccharomyces cerevisiae IFST062013, a potential probiotic," BMC Complementary and Alternative Medicine, vol. 17, no. 1, p. 64, 2017.

[17] A. A. Baker, E. Davis, J. D. Spencer, R. Moser, and T. Rehberger, "The effect of a Bacillus-based direct-fed microbial supplemented to sows on the gastrointestinal microbiota of their neonatal piglets," Journal of Animal Science, vol. 91, no. 7, pp. 3390-3399, 2013.

[18] D. Song, X. Li, Y. Cheng et al., "Effects of supplementing sow diets with Saccharomyces cerevisiae refermented sorghum dried distiller's grains with solubles from late gestation to weaning on the performance of sows and progeny," Journal of Animal Science, vol. 95, no. 5, pp. 2025-2031, 2017.

[19] R. Lan and I. Kim, "Enterococcus faecium supplementation in sows during gestation and lactation improves the performance of sucking piglets," Veterinary Medicine and Science, vol. 6, no. 1, pp. 92-99, 2020.

[20] Y. Li, H. Liu, L. Zhang et al., "Maternal dietary fiber composition during gestation induces changes in offspring antioxidative capacity, inflammatory response, and gut microbiota in a sow model," International Journal of Molecular Sciences, vol. 21, no. 1, p. 31, 2020.

[21] C. Ma, W. Zhang, Q. Gao et al., "Dietary synbiotic alters plasma biochemical parameters and fecal microbiota and metabolites in sows," Journal of Functional Foods, vol. 75, article 104221, 2020.

[22] S. J. Koopmans and T. Schuurman, "Considerations on pig models for appetite, metabolic syndrome and obese type 2 diabetes: from food intake to metabolic disease," European Journal of Pharmacology, vol. 759, pp. 231-239, 2015.

[23] J. Yang, L. Dai, Q. Yu, and Q. Yang, "Histological and anatomical structure of the nasal cavity of Bama minipigs," PLoS One, vol. 12, no. 3, article e0173902, 2017. 
[24] K. Wang, Q. Zhu, X. Kong et al., "Dietary probiotics or synbiotics supplementation during gestation, lactation, and nursery periods modifies colonic microbiota, antioxidant capacity, and immune function in weaned piglets," Frontiers in Veterinary Science, vol. 7, p. 597832, 2020.

[25] M. Yu, C. Zhang, Y. Yang et al., "Long-term effects of early antibiotic intervention on blood parameters, apparent nutrient digestibility, and fecal microbial fermentation profile in pigs with different dietary protein levels," Journal of Animal Science and Biotechnology, vol. 9, no. 1, pp. 175-186, 2018.

[26] H. Maeda, C. Fujimoto, Y. Haruki et al., "Quantitative realtime PCR using TaqMan and SYBR green for Actinobacillus actinomycetemcomitans, Porphyromonas gingivalis, Prevotella intermedia, tetQ gene and total bacteria," FEMS Immunology and Medical Microbiology, vol. 39, no. 1, pp. 81-86, 2003.

[27] X. Guo, X. Xia, R. Tang, J. Zhou, H. Zhao, and K. Wang, "Development of a real-time PCR method for Firmicutes and Bacteroidetes in faeces and its application to quantify intestinal population of obese and lean pigs," Letters in Applied Microbiology, vol. 47, no. 5, pp. 367-373, 2008.

[28] T. Matsuki, K. Watanabe, J. Fujimoto, T. Takada, and R. Tanaka, "Use of $16 \mathrm{~S}$ rRNA gene-targeted group-specific primers for real-time PCR analysis of predominant bacteria in human feces," Applied and Environmental Microbiology, vol. 70, no. 12, pp. 7220-7228, 2004.

[29] X. W. Huijsdens, R. K. Linskens, M. Mak, S. G. Meuwissen, C. M. Vandenbroucke-Grauls, and P. H. Savelkoul, "Quantification of bacteria adherent to gastrointestinal mucosa by realtime PCR," Journal of Clinical Microbiology, vol. 40, no. 12, pp. 4423-4427, 2002.

[30] E. Khafipour, S. Li, J. C. Plaizier, and D. O. Krause, "Rumen microbiome composition determined using two nutritional models of subacute ruminal acidosis," Applied and Environmental Microbiology, vol. 75, no. 22, pp. 7115-7124, 2009.

[31] A. W. Walker, J. Ince, S. H. Duncan et al., "Dominant and dietresponsive groups of bacteria within the human colonic microbiota," The ISME Journal, vol. 5, no. 2, pp. 220-230, 2011.

[32] M. A. K. Azad, G. Liu, P. Bin et al., "Sulfur-containing amino acid supplementation to gilts from late pregnancy to lactation altered offspring's intestinal microbiota and plasma metabolites," Applied Microbiology and Biotechnology, vol. 104, no. 3, pp. 1227-1242, 2020.

[33] C. N. Hsu and Y. L. Tain, "The good, the bad, and the ugly of pregnancy nutrients and developmental programming of adult disease," Nutrients, vol. 11, no. 4, p. 894, 2019.

[34] Y.Sanz, "Gut microbiota and probiotics in maternal and infant health," The American Journal of Clinical Nutrition, vol. 94, 6 Suppl, pp. 2000S-2005S, 2011.

[35] Y. Wang, Y. Wu, Y. Wang et al., "Antioxidant properties of probiotic bacteria," Nutrients, vol. 9, no. 5, p. 521, 2017.

[36] R. Mittler, S. Vanderauwera, N. Suzuki et al., "ROS signaling: the new wave?," Trends in Plant Science, vol. 16, no. 6, pp. 300-309, 2011.

[37] A. G. Pirinccioglu, D. Gokalp, M. Pirinccioglu, G. Kizil, and M. Kizil, "Malondialdehyde (MDA) and protein carbonyl (PCO) levels as biomarkers of oxidative stress in subjects with familial hypercholesterolemia," Clinical Biochemistry, vol. 43, no. 15, pp. 1220-1224, 2010.

[38] O. M. Ighodaro and O. A. Akinloye, "First line defence antioxidants-superoxide dismutase (SOD), catalase (CAT) and glutathione peroxidase (GPX): their fundamental role in the entire antioxidant defence grid," Alexandria Journal of Medicine, vol. 54, pp. 287-293, 2019.

[39] J. Degroote, W. Wang, H. Vergauwen, S. De Smet, C. Van Ginneken, and J. Michiels, "Impact of a dietary challenge with peroxidized oil on the glutathione redox status and integrity of the small intestine in weaned piglets," Animal, vol. 13, no. 8, pp. 1641-1650, 2019.

[40] J. M. Allaire, S. M. Crowley, H. T. Law, S. Y. Chang, H. J. Ko, and B. A. Vallance, "The intestinal epithelium: central coordinator of mucosal immunity," Trends in Immunology, vol. 39, no. 9, pp. 677-696, 2018.

[41] Y. Liu, F. Chen, J. Odle et al., "Fish oil enhances intestinal integrity and inhibits TLR4 and NOD2 signaling pathways in weaned pigs after LPS challenge," The Journal of Nutrition, vol. 142, no. 11, pp. 2017-2024, 2012.

[42] X. L. Gu, H. Li, Z. H. Song, Y. N. Ding, X. He, and Z. Y. Fan, "Effects of isomaltooligosaccharide and Bacillus supplementation on sow performance, serum metabolites, and serum and placental oxidative status," Animal Reproduction Science, vol. 207, pp. 52-60, 2019.

[43] L. Nikniaz, R. Mahdavi, A. Ostadrahimi, M. A. Hejazi, and A. M. Vatankhah, "Effects of synbiotic supplementation on total antioxidant capacity of human breastmilk," Breastfeeding Medicine, vol. 8, no. 2, pp. 217-222, 2013.

[44] X. L. Chen, G. Dodd, S. Thomas et al., "Activation of Nrf2/ARE pathway protects endothelial cells from oxidant injury and inhibits inflammatory gene expression," American Journal of Physiology. Heart and Circulatory Physiology, vol. 290, no. 5, pp. H1862-H1870, 2006.

[45] M. Watford, P. Lund, and H. A. Krebs, "Isolation and metabolic characteristics of rat and chicken enterocytes," The Biochemical Journal, vol. 178, no. 3, pp. 589-596, 1979.

[46] K. A. Moore and I. R. Lemischka, "Stem cells and their niches," Science, vol. 311, no. 5769, pp. 1880-1885, 2006.

[47] A. H. Bhat, K. B. Dar, S. Anees et al., "Oxidative stress, mitochondrial dysfunction and neurodegenerative diseases; a mechanistic insight," Biomedicine \& Pharmacotherapy, vol. 74, pp. 101-110, 2015.

[48] M. I. Ekstrand, M. Falkenberg, A. Rantanen et al., "Mitochondrial transcription factor A regulates mtDNA copy number in mammals," Human Molecular Genetics, vol. 13, no. 9, pp. 935944, 2004.

[49] W. Tunapong, N. Apaijai, S. Yasom et al., "Chronic treatment with prebiotics, probiotics and synbiotics attenuated cardiac dysfunction by improving cardiac mitochondrial dysfunction in male obese insulin-resistant rats," European Journal of Nutrition, vol. 57, no. 6, pp. 2091-2104, 2018.

[50] K. Veljović, M. Dinić, J. Lukić et al., "Promotion of early gut colonization by probiotic intervention on microbiota diversity in pregnant sows," Frontiers in Microbiology, vol. 8, p. 2028, 2017.

[51] S. Michael, G. Claudia, J. Y. Rose, I. Peter, and A. V. Jon, "Administration of oral probiotic bacteria to pregnant women causes temporary infantile colonization," Journal of Pediatric Gastroenterology and Nutrition, vol. 38, pp. 293-297, 2004.

[52] N. F. Krebs, J. E. Westcott, N. Butler, C. Robinson, and K. M. Hambidge, "Meat as a first complementary food for breastfed infants: feasibility and impact on zinc intake and status," Journal of Pediatric Gastroenterology and Nutrition, vol. 42, no. 2, pp. 207-214, 2006.

[53] L. V. Hooper, T. Midtvedt, and J. I. Gordon, "How hostmicrobial interactions shape the nutrient environment of the 
mammalian intestine," Annual Review of Nutrition, vol. 22, no. 1, pp. 283-307, 2002.

[54] H. Ye, J. Liu, P. Feng, W. Zhu, and S. Mao, "Grain-rich diets altered the colonic fermentation and mucosa-associated bacterial communities and induced mucosal injuries in goats," Scientific Reports, vol. 6, no. 1, p. 20329, 2016.

[55] S. Doron and S. L. Gorbach, "Probiotics: their role in the treatment and prevention of disease," Expert Review of AntiInfective Therapy, vol. 4, no. 2, pp. 261-275, 2006.

[56] M. I. Alvarez-Olmos and R. A. Oberhelman, "Probiotic agents and infectious diseases: a modern perspective on a traditional therapy," Clinical Infectious Diseases, vol. 32, no. 11, pp. 1567-1576, 2001.

[57] Y. Qiao, J. Sun, Y. Ding, G. Le, and Y. Shi, "Alterations of the gut microbiota in high-fat diet mice is strongly linked to oxidative stress," Applied Microbiology and Biotechnology, vol. 97, no. 4, pp. 1689-1697, 2013. 